PROCEEDINGS OF THE

AMERICAN MATHEMATICAL SOCIETY

Volume 133, Number 9, Pages 2697-2703

S 0002-9939(05)08152-9

Article electronically published on April 25, 2005

\title{
ON THE PROXIMINALITY OF THE UNIT BALL OF PROXIMINAL SUBSPACES IN BANACH SPACES: A COUNTEREXAMPLE
}

FATHI B. SAIDI

(Communicated by Jonathan M. Borwein)

\begin{abstract}
A known, and easy to establish, fact in Best Approximation Theory is that, if the unit ball of a subspace $G$ of a Banach space $X$ is proximinal in $X$, then $G$ itself is proximinal in $X$. We are concerned in this article with the reverse implication, as the knowledge of whether the unit ball is proximinal or not is useful in obtaining information about other problems. We show, by constructing a counterexample, that the answer is negative in general.
\end{abstract}

\section{INTRODUCTION}

Let $X$ be a Banach space with norm $\|$.$\| and distance d(.,$.$) . We need the$ following definition:

Definition 1. A nonempty subset $G$ of the Banach space $X$ is said to be $\|\|-$. proximinal (or proximinal) in $X$ if for every $x \in X$ there exists $g_{x} \in G$ such that

$$
\left\|x-g_{x}\right\|=d(x, G):=\inf \{\|x-g\|: g \in G\} .
$$

A point $g_{x} \in G$ that satisfies Eq. (1.1) is called a best approximation of $x$ from $G$. The set of best approximations from $G$ of a point $x \in X$ is denoted by $\pi_{G}(x)$ or $\pi(x)$. If every point $x \in X$ admits a unique best approximation from $G$, then $G$ is said to be Chebyshev in $X$.

The concept of best approximation is one of the most important in approximation theory. It plays an important role in many areas of science. For this reason, a lot of work has been devoted to the study of proximinality of subspaces of Banach spaces. We refer the reader to [2], [5], and the references cited there for a great deal of literature on the theory of best approximation.

In connection with the proximinality of subspaces of Banach spaces, one encounters the problem of characterization of the relation, if any, that exists between the proximinality of the subspace and the proximinality of its unit ball. It is easy to show that if the unit ball $B_{G}$ of the subspace $G$ of $X$ is proximinal in $X$, then $G$ itself is proximinal in $X$. We are concerned here with the reverse implication. In other words, our aim in this paper is to investigate the following problem.

Received by the editors April 28, 2004.

2000 Mathematics Subject Classification. Primary 41A65, 41A50; Secondary 41A52, 41A30.

Key words and phrases. Proximinality, best approximation, approximation, Banach spaces, unit ball, counterexample, example, renorming.

(C) 2005 American Mathematical Society Reverts to public domain 28 years from publication 2697 
Problem 1. Given a Banach space $X$ and given a subspace $G$ of $X$, is the unit ball $B_{G}$ of $G$ proximinal in $X$ whenever the subspace $G$ is proximinal in $X$ ?

One may be inclined to think that, if $G$ is a proximinal subspace of $X$, then, for any $x \in X$, the projection of the set $\pi_{G}(x)$ onto the unit ball $B_{G}$ of $G$ contains a best approximation of $x$ from $B_{G}$, hence the answer to Problem 1 is affirmative. By projection of an element $g \in G$ onto $B_{G}$ we mean $g$ if $g \in B_{G}$ and $g /\|g\|$ if $g \notin B_{G}$. This leads us to the investigation of the following problem.

Problem 2. Given a proximinal subspace $G$ of a Banach space $X$, is it true that for every $x \in X$ we have

$$
\pi_{B_{G}}(x) \cap P_{B_{G}}\left(\pi_{G}(x)\right) \neq \emptyset,
$$

where, for every $g \in G, P_{B_{G}}(g)=g$ if $g \in B_{G}$ and $P_{B_{G}}(g)=g /\|g\|$ if $g \notin B_{G}$ ?

Problem 2 was addressed in [4]. There the author showed that the answer is negative in general, even when $B_{G}$ is also proximinal in $X$. Moreover, it was shown that if all closed subspaces $G$ of $X$ are Chebyshev in $X$ (equivalently, if $X$ is reflexive and strictly convex, 5, Corollary 3.4, page 111]) and if the answer to Problem 2 was affirmative for all closed subspaces $G$ of a fixed finite dimension $n$ or for all closed subspaces $G$ of a fixed finite codimension $(n-1), 2 \leq n<\operatorname{dim}(X)$, then $X$ is isometric to a Hilbert space. But still, there remains the question of whether the unit ball $B_{G}$ is proximinal in $X$ whenever $G$ is proximinal in $X$. We show in this paper, by constructing a counterexample, that the answer to Problem 1 is negative in general.

Finally, we point out that it is important to know whether the unit ball $B_{G}$ is proximinal in $X$ or not, as this information may be used to obtain results about other problems. For instance, in [3] and [1] the authors use the proximinality of the unit ball $B_{G}$ of the subspace $G$ of $X$ to obtain results on the proximinality of $L^{p}(S, G)$ in $L^{p}(S, X)$, where $S$ is a finite measure space and $L^{p}(S, X)$ is the usual $L^{p}$-space of $p$-Bochner-integrable functions defined on $S$ with values in $X$.

\section{The Counterexample}

In any Banach space $E$ with norm denoted by $\|$.$\| , the ball centered at e \in E$ and radius $r \in[0, \infty)$ is denoted by $B_{E}^{\|\cdot\|}(e, r)$, or simply by $B_{E}(e, r)$ when there is no confusion, and is given by

$$
B_{E}^{\|. \cdot\|}(e, r)=B_{E}(e, r)=\{x \in E:\|x-e\| \leq r\} .
$$

The unit ball in $E$ is also denoted by $B_{E}^{\| .||}$or by $B_{E}$,

$$
B_{E}^{\|. \cdot\|}=B_{E}=\{x \in E:\|x\| \leq 1\},
$$

while the distance in $E$ is denoted by $d_{\|. .\|}(.,$.$) or by d(.,$.$) . Finally, the set of real$ numbers is denoted by $\mathbb{R}$.

We now start the construction of our counterexample. Let $Z$ be a one-dimensional real Banach space and let $G$ be a real Banach space that contains a nonproximinal hyperplane $H$ (note that every nonreflexive Banach space $G$ satisfies this property, 5, page 100]). We denote the norms on $G$ and $Z$ by $\|\cdot\|_{G}$ and $\|\cdot\|_{Z}$, respectively.

Let $X$ be the Banach space given by the $l^{1}$-sum of $G$ and $Z$,

$$
X:=G \oplus_{1} Z \text {. }
$$


In other words, $X$ is the direct sum of $G$ with $Z$, and the norm on $X$ is the $l^{1}$-norm $\|.\|_{1}$ defined by

$$
\|x\|_{1}:=\|g\|_{G}+\|z\|_{Z},
$$

for every $x:=g+z$ where $g \in G$ and $z \in Z$. Note that $\|\cdot\|_{1}=\|\cdot\|_{G}$ on $G$ and $\|\cdot\|_{1}=\|\cdot\|_{Z}$ on $Z$.

Now, let $z_{o} \in Z$ be fixed, $\left\|z_{o}\right\|_{Z}=1$. Then the unit ball in $X$ is the convex hull of the set $\left\{z_{o},-z_{o}, B_{G}\right\}$, i.e.

$$
B_{X}:=B_{X}^{\|\cdot\|_{1}}=\operatorname{co}\left\{z_{o},-z_{o}, B_{G}\right\} .
$$

Note that one can easily check that $B_{X}$ is indeed closed in $X$. Since $H$ is nonproximinal in $G$, there exists $\bar{g} \in G$ such that $d(\bar{g}, H)=1$ and

$$
B_{G}(\bar{g}, 1) \cap H=\emptyset .
$$

Let $\bar{H}$ be the manifold given by $\bar{H}:=\bar{g}+H$. Then we have

$$
d(0, \bar{H})=1 \text { and } B_{G} \cap \bar{H}=\emptyset .
$$

Now let $A$ be the subset of $\bar{H}$ defined by $A:=B_{G}(0,2) \cap \bar{H}$ and let $a \in A$ be fixed. Consider the subset $R$ of $H$ given by

$$
R:=A-a .
$$

Then $0 \in R$ since $a \in A$. Since $R$ is a bounded subset of $G$, there exists $\gamma \in(0, \infty)$ such that

Let $D:=\frac{1}{\gamma} R-z_{o}$ and let

$$
\frac{1}{\gamma}(R \cup(-R)) \subset B_{G}
$$

$$
K:=c l_{X}\left(\operatorname{co}\left\{D, B_{G},-D\right\}\right),
$$

where $c_{X}(M)$ denotes the closure in $X$ of the subset $M$ of $X$. Since $0 \in R$, it follows that $z_{o}$ and $-z_{o}$ are both in $K$ and, consequently, that $B_{X} \subset K$. This, together with the fact that $K$ is bounded in $X$, implies that there exists $\rho \in(0, \infty)$ such that

$$
B_{X} \subset K \subset B_{X}(0, \rho) .
$$

It follows that the Minkowski functional associated with $K$ defines a norm $\|$.$\| on$ $X$ that is equivalent to the $l^{1}$-norm $\|.\|_{1}$.

Note that the unit ball in $(X,\|\|$.$) is given by K$. Hence

$$
B_{X}^{\|\cdot \cdot\|}=K
$$

To avoid confusion, we substitute the letter $K$ for the letter $B$ when referring to balls in $X$ corresponding to the new norm $\|$.$\| . Thus, for instance,$

$$
K_{X}:=B_{X}^{\|\cdot \mid\|}=K \text { and } K_{X}(x, r):=B_{X}^{\|\cdot\|}(x, r) .
$$

Our goal in the remainder of this paper is to show that $G$ is $\|$.$\| -proximinal in$ $X$ while the unit ball $K_{G}$ of $G$ is not ||.||-proximinal in $X$.

We start with the following remark which follows directly from the definitions of $K$ and $\|\cdot\|_{1}$.

Remark 1. The norm on $G$ induced by the new norm $\|$.$\| is the same as the old$ norm $\|.\|_{G}$. In other words, we have

$$
K_{G}:=K \cap G=B_{G} .
$$


For each $x \in X$, let $\pi(x)$ denote the set of best approximations of $x$ from $G$ with respect to the new norm $\|$.$\| ,$

$$
\pi(x):=\left\{y \in G:\|x-y\|=d_{\|. \mid\|}(x, G)\right\} .
$$

Our next lemma shows that $G$ is $\|$.$\| -proximinal in X$ while explicitly providing the set of best approximations of an arbitrary $x:=g+\lambda z_{o} \in X$, where $g \in G$.

Lemma 1. The following statements hold true:

(i) For every $g \in G$ and every $\lambda \in \mathbb{R}$, we have

$$
K\left(g+\lambda z_{o}, \tau\right) \cap G=\left\{\begin{array}{lll}
\emptyset & \text { if } & \tau<\lambda, \\
g+\frac{\lambda}{\gamma} R=g+\lambda\left(D+z_{o}\right) & \text { if } & \tau=\lambda .
\end{array}\right.
$$

(ii) For every $g \in G$ and every $\lambda \in \mathbb{R}$, we have

$$
\pi\left(g+\lambda z_{o}\right)=g+\frac{\lambda}{\gamma} R=g+\lambda\left(D+z_{o}\right)
$$

and

$$
d_{|| .||}\left(g+\lambda z_{o}, G\right)=\lambda
$$

Hence $G$ is $\|$.$\| -proximinal in X$.

Proof. First, note that part (ii) follows directly from part (i).

Now, clearly we have, for every $g \in G$ and every $\lambda \in \mathbb{R}$,

$$
K\left(g+\lambda z_{o}, \tau\right) \cap G=g+\lambda\left(K\left(z_{o}, \frac{\tau}{\lambda}\right) \cap G\right) .
$$

Therefore Eq. (2.2) is equivalent to

$$
K\left(z_{o}, T\right) \cap G=\left\{\begin{array}{lll}
\emptyset & \text { if } & T<1, \\
\frac{1}{\gamma} R=D+z_{o} & \text { if } & T=1 .
\end{array}\right.
$$

If $T=0$, then $K\left(z_{o}, T\right)=\left\{z_{o}\right\} \notin G$, hence $K\left(z_{o}, T\right) \cap G=\emptyset$.

Now, suppose that $K\left(z_{o}, T\right) \cap G \neq \emptyset$ for some $T>0$ and let $h \in K\left(z_{o}, T\right) \cap G$ be fixed. Then

$$
\frac{\left(h-z_{o}\right)}{T} \in K:=\operatorname{cl}_{X}\left(\operatorname{co}\left\{D, B_{G},-D\right\}\right) .
$$

It follows, since $D=(1 / \gamma) R-z_{o}$, that there exist sequences $\left\{r_{n}\right\},\left\{r_{n}^{-}\right\}$, and $\left\{h_{n}\right\}$ in $(1 / \gamma) R,(1 / \gamma)(-R)$, and $B_{G}$ respectively such that

$$
\frac{\left(h-z_{o}\right)}{T}=\lim \left(\alpha_{n} r_{n}+\beta_{n} r_{n}^{-}+\delta_{n} h_{n}+\left(\beta_{n}-\alpha_{n}\right) z_{o}\right),
$$

where $\left\{\alpha_{n}\right\},\left\{\beta_{n}\right\}$, and $\left\{\delta_{n}\right\}$ are sequences in $[0,1]$ satisfying

$$
\alpha_{n}+\beta_{n}+\delta_{n}=1 .
$$

This implies, by the definition of $\|\cdot\|_{1}$, that $\lim \left(\beta_{n}-\alpha_{n}\right)=-1 / T$. Hence $\left(\alpha_{n}-\beta_{n}\right) \neq 0$ for large $n$ and

$$
T=\lim \left(\frac{1}{\alpha_{n}-\beta_{n}}\right) \geq 1,
$$

where the inequality follows from the fact that $\alpha_{n}$ and $\beta_{n}$ are both in the interval $[0,1]$. Therefore

$$
K\left(z_{o}, T\right) \cap G=\emptyset \text { if } T<1 .
$$


On the other hand, if $T=1$, then we obtain that $\lim \left(\alpha_{n}-\beta_{n}\right)=1$ and, consequently, since $\alpha_{n}$ and $\beta_{n}$ are both in $[0,1]$ and since $\alpha_{n}+\beta_{n}+\delta_{n}=1$, that

$$
\lim \alpha_{n}=1 \text { and } \lim \beta_{n}=\lim \delta_{n}=0 .
$$

It follows, since $\left\{r_{n}^{-}\right\}$and $\left\{h_{n}\right\}$ are bounded $\left(r_{n}^{-} \in \frac{1}{\gamma}(-R) \subset B_{G}\right.$ and $\left.h \in B_{G}\right)$, that $\lim \beta_{n} r_{n}^{-}=\lim \delta_{n} h_{n}=0$ and, consequently, that

$$
h=\lim r_{n} .
$$

But $\left\{r_{n}\right\} \subset(1 / \gamma) R$, which is closed. Therefore $h \in(1 / \gamma) R$ and, consequently,

$$
\left(K\left(z_{o}, 1\right) \cap G\right) \subset \frac{1}{\gamma} R .
$$

The reverse inclusion follows from the fact that $\left((1 / \gamma) R-z_{o}\right) \subset K$, hence $(1 / \gamma) R$ $\subset K\left(z_{o}, 1\right)$, and the fact that $(1 / \gamma) R \subset G$. Therefore

$$
K\left(z_{o}, 1\right) \cap G=\frac{1}{\gamma} R .
$$

This completes the proof of Eq. (2.3) and, consequently, of Eq. (2.2). The proof of the lemma is now complete.

It remains to show that the unit ball $K_{G}$ of $G$ is not $\|$.$\| -proximinal in X$. Indeed, we have:

Lemma 2. The unit ball $K_{G}$ of $G$ is not $\|$.$\| -proximinal in X$.

Proof. First note that, by Remark 1, $K_{G}=B_{G}$ and, consequently,

$$
d_{|| \cdot \mid}=d_{\|\mid\|_{1}}=d \text { on } G \times G \text {. }
$$

By Lemma 1 and the definition of $R$, we have, for every $g \in G$ and every $\lambda \in \mathbb{R}$,

$$
\pi\left(g+\lambda z_{o}\right)=g+\frac{\lambda}{\gamma} R=g-\frac{\lambda}{\gamma} a+\frac{\lambda}{\gamma} A .
$$

Let $\bar{x}$ be the fixed element of $X$ defined by

$$
\bar{x}:=a+\gamma z_{o} .
$$

Then we have, by Eq. (2.5) and Lemma 1

$$
\pi(\bar{x})=K(\bar{x}, \gamma) \cap G=A \text { and } d_{\|\cdot\|}(\bar{x}, G)=\gamma .
$$

It follows, by Eqs. (2.1) and (2.4) and by the definition of $A$, that

$$
d\left(\pi(\bar{x}), B_{G}\right)=d\left(A, B_{G}\right)=d\left(\bar{H}, B_{G}\right)=0
$$

and

$$
\pi(\bar{x}) \cap B_{G}=A \cap B_{G}=\emptyset .
$$

This together with Eq. (2.6) imply that $d\left(\pi(\bar{x}), B_{G}\right)=0$ and, consequently,

$$
d_{\||.|}\left(\bar{x}, B_{G}\right)=d_{\|. \mid\|}(\bar{x}, G)=\gamma \text { and } K(\bar{x}, \gamma) \cap B_{G}=\emptyset .
$$

This implies that $\bar{x}$ has no best approximation from $B_{G}$. It follows, since $K_{G}=B_{G}$, that $K_{G}$ is not $\|$.$\| -proximinal in X$, which completes the proof of the lemma.

In summary, we have proved the following theorem, which represents our main result. 
Theorem 1. Let $G$ be a real Banach space that contains a nonproximinal hyperplane, let $Z$ be a one-dimensional real Banach space, and let $X$ be the Banach space given by the $l^{1}$-sum of $G$ and $Z, X:=G \oplus_{1} Z$. Then there exists a norm $\|$.$\| on X$ equivalent to the $l^{1}$-norm $\|.\|_{1}$ such that $G$ is $\|$.$\| -proximinal in X$, while the unit ball $B_{G}^{\|.\|}$of $G$ is not $\|$.$\| -proximinal in X$.

Since every nonreflexive Banach space contains a nonproximinal hyperplane, [5] page 100], we immediately obtain the following two corollaries:

Corollary 1. Let $G$ be a nonreflexive real Banach space, let $Z$ be a real Banach space of dimension one, and let $X$ be the Banach space given by the $l^{1}$-sum of $G$ and $Z, X:=G \oplus_{1} Z$. Then there exists a norm $\|$.$\| on X$ equivalent to the $l^{1}$ norm $\|.\|_{1}$ such that $G$ is $\|$.$\| -proximinal in X$ while the unit ball $B_{G}^{\|\cdot\|}$ of $G$ is not \|.\|-proximinal in $X$.

Corollary 2. There exists a Banach space $X$ that contains a proximinal subspace $G$ whose unit ball $B_{G}$ is nonproximinal in $X$.

We conclude by showing that it was necessary to renorm $X$ in order to obtain a counterexample. Indeed, the unit ball $B_{G}$ of $G$ is $\|.\|_{1}$-proximinal in $X$, as the following proposition shows.

Proposition 1. Let $G$ be a real Banach space, let $Z$ be a one-dimensional real Banach space, and let $X$ be the Banach space given by the $l^{1}$-sum of $G$ and $Z$, $X:=G \oplus_{1} Z$. Then $G$ and its unit ball $B_{G}$ are both $\|.\|_{1}$-proximinal in $X$.

Proof. Let $x:=g_{x}+z_{x} \in X$ be fixed, $g_{x} \in G$ and $z_{x} \in Z$. Clearly we have, for every $g \in G$,

$$
\|x-g\|_{1}=\left\|g_{x}-g\right\|_{G}+\left\|z_{x}\right\|_{Z} \geq\left\|z_{x}\right\|_{Z}=\left\|x-g_{x}\right\|_{1} .
$$

Therefore $g_{x}$ is a best approximation of $x$ from $G$ with respect to $\|\cdot\|_{1}$ and, consequently,

$$
G \text { is }\|\cdot\|_{1} \text {-proximinal in } X \text {. }
$$

In fact, $g_{x}$ is the unique best approximation of $x$ from $G$ with respect to $\|.\|_{1}$, hence $G$ is Chebyshev in $X$.

Now, if $g_{x} \in B_{G}$, then $g_{x}$ is also a best approximation of $x$ from $B_{G}$ with respect to $\|.\|_{1}$. Therefore, suppose that $g_{x} \notin B_{G}$. Then we have

$$
\left\|g_{x}-\frac{g_{x}}{\left\|g_{x}\right\|}\right\|_{G}=\inf \left\{\left\|g_{x}-g\right\|_{G}: g \in B_{G}\right\} .
$$

It follows that

$$
\begin{aligned}
\left\|x-\frac{g_{x}}{\left\|g_{x}\right\|}\right\|_{1} & =\left\|g_{x}-\frac{g_{x}}{\left\|g_{x}\right\|}\right\|_{G}+\left\|z_{x}\right\|_{Z} \\
& \leq\left\|g_{x}-g\right\|_{G}+\left\|z_{x}\right\|_{Z}=\|x-g\|_{1},
\end{aligned}
$$

for all $g \in B_{G}$. Hence $g_{x} /\left\|g_{x}\right\|$ is a best approximation of $x$ from $B_{G}$ with respect to $\|.\|_{1}$. Therefore in all cases $x$ has a best approximation from $B_{G}$ with respect to $\|\cdot\|_{1}$ and, consequently, $B_{G}$ is $\|.\|_{1}$-proximinal in $X$. This completes the proof of the proposition. 


\section{REFERENCES}

[1] W. Light, Proximinality in $L_{p}(S, X)$, Rocky Mountain J. Math. 19 (1989), no.1, 251-259. MR.1016178 (91a:41037)

[2] W. A. Light and E. W. Cheney, "Approximation theory in tensor product spaces", Lecture Notes in Mathematics, Vol. 1169, Springer-Verlag, Berlin/New York, 1985. MR0817984 (87g:41064)

[3] Fathi B. Saidi, On the smoothness of the metric projection and its application to proximinality in $L^{p}(S, X)$, J. Approx. Theory 83 (1995), 205-319. MR.1357587 (96h:41042)

[4] Fathi B. Saidi, Subdifferentials and approximation theory, Numer. Funct. Anal. and Optimiz. 16(5\&6) (1995), 765-783. MR 1341111 (97a:41036)

[5] I. Singer, Best approximation in normed linear spaces by elements of linear subspaces, SpringerVerlag, New York, 1970. MF0270044 (42:4937)

Mathematics Division, University of Sharjah, P. O. Box 27272, Sharjah, United Arab EMIRATES

E-mail address: fsaidi@sharjah.ac.ae 